\title{
Magnetic and Electronic Quantum Criticality in $\mathrm{YbRh}_{2} \mathrm{Si}_{2}$
}

\author{
S. Friedemann • T. Westerkamp • M. Brando • \\ N. Oeschler • P. Gegenwart • C. Krellner • \\ C. Geibel • S. MaQuilon · Z. Fisk • F. Steglich • \\ S. Wirth
}

Received: 8 April 2010 / Accepted: 7 July 2010 / Published online: 28 July 2010

(C) Springer Science+Business Media, LLC 2010

\begin{abstract}
The unconventional nature of the quantum criticality in $\mathrm{YbRh}_{2} \mathrm{Si}_{2}$ is highlighted on the basis of magnetoresistivity and susceptibility measurements. Results obtained under chemical pressure realized by isoelectronic substitution on the rhodium site are presented. These results illustrate the position of the $T^{\star}$-line associated with a breakdown of the Kondo effect near the antiferromagnetic instability in the low-temperature phase diagram. Whereas at zero temperature the Kondo breakdown and the antiferromagnetic quantum critical point coincide in the proximity of the stoichiometric compound, they are seen to be detached under chemical pressure: For positive chemical pressure the magnetically ordered phase extends beyond the $T^{\star}(B)$-line. For sufficiently high negative pressure the $T^{\star}(B)$-line is separated from the magnetically ordered phase. From our detailed analysis we infer that the coincidence is retained at small iridium concentrations, i.e., at small negative chemical pressure. We outline further measurements which may help to clarify the detailed behavior of the two instabilities.
\end{abstract}

Keywords Heavy fermion metals · Quantum criticality · Kondo breakdown · Chemical pressure

\footnotetext{
S. Friedemann · T. Westerkamp · M. Brando · N. Oeschler · P. Gegenwart · C. Krellner · C. Geibel · F. Steglich · S. Wirth $(\bowtie)$

Max Planck Institute for Chemical Physics of Solids, Nöthnitzer Str. 40, 01187 Dresden, Germany e-mail: wirth@cpfs.mpg.de

S. Friedemann

e-mail: Sven.Friedemann@cpfs.mpg.de

P. Gegenwart

I. Physics Institute, University of Göttingen, Friedrich-Hund-Platz 1, 37077 Göttingen, Germany

S. MaQuilon · Z. Fisk

Department of Physics and Astronomy, University of California, Irvine, CA 92697-4575, USA
} 


\section{Introduction}

Quantum criticality occurs when a continuous phase transition is suppressed to zero temperature by means of an external tuning parameter. It provides a route to investigate newly emerging behavior arising from strong correlations of modes of a classical order parameter and inherently quantum modes [1]. Heavy-fermion systems adopt a key role in the study of quantum criticality [2]. These systems often display antiferromagnetic order at low temperatures. Pressure, chemical composition and magnetic field are utilized to tune the corresponding transition to zero temperature. The physical properties of these heavy-fermion metals are dominated by the hybridization of $f$ electrons (provided by rare earth or actinide elements) with conduction electrons. On the one hand, this hybridization can give rise to the Kondo effect and resulting new, composite quasiparticles. The latter are often found to obey the standard LandauFermi-liquid picture at lowest temperatures but with strongly enhanced Landau parameters reflected, e.g., by a renormalized mass of up to the order of 1000 bare electron masses. On the other hand, in heavy-fermion metals also magnetic order may arise from the indirect exchange between neighboring $f$-sites mediated by the conduction electrons, the so-called Ruderman-Kitel-Kasuya-Yosida (RKKY) interaction.

For the materials of interest here, the magnetic order competes with the Kondo effect. The ability to tune the relative strength of the associated energies allows one to adjust the ground state in these materials. This is possible in a controlled fashion as both the Kondo and the RKKY temperatures, $T_{\mathrm{K}}$ and $T_{\mathrm{RKKY}}$ depend differently on the exchange interaction between local moments and conduction electrons [3]: $T_{\mathrm{RKKY}}$ exhibits a quadratic form, whereas $T_{\mathrm{K}}$ follows an exponential one. This gives rise to two different regimes: In the weak coupling regime a magnetic ground state originates in the dominance of the RKKY interaction. In the strong coupling regime, the Kondo effect provides a paramagnetic ground state. Right at the continuous transition, i.e., at the point where the two just balance each other, the magnetic order is suppressed to zero temperature and a quantum critical point (QCP) emerges. One way to control the exchange interaction, and thereby tuning a material to its QCP, is to increase the overlap of the $f$-electron and conduction-electron wave functions. This might be realized by changing the unit-cell volume through either pressure or an adjustment of the chemical composition. Alternatively, a magnetic field can be utilized to modify the antiferromagnetic ground state.

Two different scenarios have been developed for the theoretical description of QCPs in antiferromagnetic heavy-fermion systems: (i) The more conventional concept is a quantum generalization of the Ginzburg-Landau-Wilson theory of finitetemperature phase transitions. It assumes that the magnetic order arises via a spindensity-wave of the heavy quasiparticles [4-6]. (ii) More recent approaches incorporate critical modes arising from a breakdown of the Kondo effect at the QCP [7-10]. A distinct difference between the two lies in the behavior of the composite quasiparticles at the QCP: For the conventional scenario, they stay intact and constitute both the magnetically ordered state via a spin-density-wave and the paramagnetic ground state. In the unconventional Kondo-breakdown scenario, by contrast, the quasiparticles disintegrate and, consequently, the Fermi-surface volume is expected to undergo a discontinuous change at the QCP. Based on this, measurements which probe the 


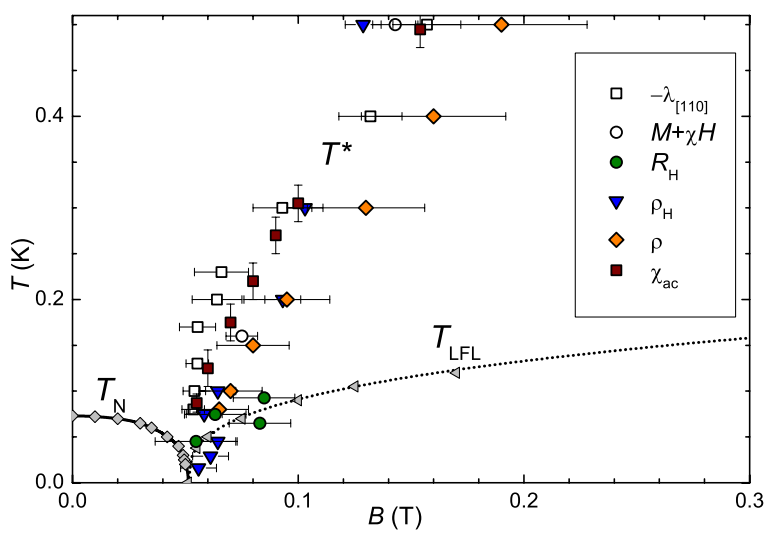

Fig. 1 (Color online) Low-temperature phase diagram of $\mathrm{YbRh}_{2} \mathrm{Si}_{2}$ after Refs. [13, 14]. Both, the Néel temperature $T_{\mathrm{N}}$ and the Landau-Fermi-liquid temperature $T_{\mathrm{LFL}}$ were obtained from resistivity measurements. They are observed as a transition and a crossover to a quadratic temperature dependence at the lowest temperatures, respectively. Signatures of the $T^{\star}$ energy scale were observed as broadened kinks in isothermal measurements of magnetostriction $\lambda$, magnetization $M$, and Hall resistivity $\rho_{\mathrm{H}}$, as crossovers in isothermal measurements of the Hall coefficient $R_{\mathrm{H}}$ and magnetoresistivity $\rho$ as well as in a maximum in the temperature dependence of the susceptibility $\chi_{\mathrm{ac}}$

volume of the Fermi sea were suggested as an experimental tool to discriminate between the two scenarios [8].

$\mathrm{YbRh}_{2} \mathrm{Si}_{2}$ adopts a key role for the investigations on the unconventional scenario. In this material, the Kondo effect leads to the formation of the composite quasiparticles below the single ion Kondo energy scale of $\approx 30 \mathrm{~K}[2,11]$. The antiferromagnetic order, however, sets in below a Néel temperature of only $T_{\mathrm{N}}=70 \mathrm{mK}$ [12]. This is illustrated in the low-temperature phase diagram in Fig. 1 which is adapted from Refs. $[13,14]$. The small ordering temperature in zero magnetic field already demonstrates that the RKKY interaction is only slightly ahead in the competition of the energy scales. Consequently, one would expect a QCP in close proximity. Indeed, the application of a small magnetic field leads to the suppression of the transition to zero temperature. At the critical field $B_{\mathrm{N}}=60 \mathrm{mT}$ applied within the basal plane the QCP is accessed [15]. If oriented along the crystallographic $c$-direction, a field of $0.7 \mathrm{~T}$ is required.

At fields above the critical field, the dominance of the Kondo effect causes the formation of a heavy Fermi liquid at sufficiently low temperatures. Here, the Sommerfeld coefficient and the quadratic-in-temperature coefficient of the resistivity are of the order of $\gamma_{0} \sim 1 \mathrm{~J}$ mole ${ }^{-1} \mathrm{~K}^{-2}$ and $A \sim 10 \mu \Omega \mathrm{cm} \mathrm{K}^{-2}$, respectively [16]. Both quantities are related to the effective mass of the quasiparticles by a direct proportionality and a proportionality to its square, respectively. In fact, the Kadowaki-Woods ratio $A / \gamma_{0}^{2}$ assumes a value observed for a wide variety of heavy-fermion metals [17] and indicates that the Landau-Fermi liquid description is indeed applicable in $\mathrm{YbRh}_{2} \mathrm{Si}_{2}$ : The strong correlations among the bare electrons lead only to minor deviations from the description in terms of non-interacting (renormalized) quasiparticles [18]. 
The presence of a QCP manifests itself in divergences of $A$ and $\gamma_{0}$ which correspond with a "slowing down" of the quasiparticles and a concomitant further enhancement of their renormalized mass [15]. In order to discriminate between the two available scenarios for its description, the Hall effect was studied while tuning the system across the QCP [14]. The measurements revealed a broadened kink in the Hall resistivity $\rho_{\mathrm{H}}$ and, equivalently, a crossover of the Hall coefficient $R_{\mathrm{H}}$ when measured isothermally as a function of magnetic field $B$. The positions of this kink/crossover are included in Fig. 1. They are seen to converge to $B_{\mathrm{N}}$ as the temperature is lowered, thereby manifesting the relation to the QCP. Moreover, the kink in $\rho_{\mathrm{H}}(B)$ and the crossover in $R_{\mathrm{H}}(B)$ sharpen as the temperature is lowered. Importantly, their widths shrink to zero in the extrapolation to zero temperature. Consequently, the Hall coefficient is inferred to exhibit an abrupt jump at the QCP. Out of this discontinuity it was concluded that the Fermi surface undergoes a severe reconstruction, thus favoring the Kondo-breakdown scenario.

Right at the position where the crossover occurs in the Hall coefficient, also other properties display pronounced signatures [13]. In particular, the magnetoresistivity $\rho(B)$ was shown to exhibit a crossover similar to that of the Hall coefficient, in accordance with theoretical predictions [19]. Furthermore, various thermodynamic properties display pronounced features across the Hall crossover line: The magnetostriction $\lambda$ as well as the magnetization $M$ exhibit a broadened kink resembling that of the Hall resistivity. The a.c. susceptibility $\chi_{\mathrm{ac}}$ is found to obey a pronounced maximum in its temperature dependence, and a crossover in its field dependence. Taking all this together, these measurements established the existence of an additional energy scale, labeled $T^{\star}(B)$, vanishing at the QCP. This is in agreement with the Kondo-breakdown scenario, in which such an energy scale is associated with the break up of the composite quasiparticles [7]. This scenario received further support by recent low-temperature measurements [20] of the field-dependent thermopower on $\mathrm{YbRh}_{2} \mathrm{Si}_{2}$.

An unexpected evolution of the energy scales emerges [21] when chemical pressure is applied to $\mathrm{YbRh}_{2} \mathrm{Si}_{2}$. For samples with small isoelectronic substitution, the volume effect shifts the magnetic transition temperature $T_{\mathrm{N}}$ and the critical field $B_{\mathrm{N}}$ (at which $T_{\mathrm{N}} \rightarrow 0$ ) as expected. By contrast, the position of the Kondo-breakdown energy scale $T^{\star}$ and its critical field $B^{\star}$ at which $T^{\star} \rightarrow 0$ is almost not altered. As a consequence, the coincidence of the Fermi-surface reconstruction and the antiferromagnetic instability (as observed in the stoichiometric compound) appears to be lifted for the samples with isoelectronic substitution.

In the following, we illustrate by a close inspection of the magnetoresistivity and the susceptibility measurements that the coincidence of the antiferromagnetic and the Kondo-breakdown critical fields $B_{\mathrm{N}}$ and $B^{\star}$ at $T \rightarrow 0$ may be present not only for the stoichiometric compound but appears to be retained even at small Ir concentrations.

\section{Magnetoresistivity}

The longitudinal magnetoresistivity was determined on single crystals of pure and substituted $\mathrm{Yb}\left(\mathrm{Rh}_{1-x} M_{x}\right)_{2} \mathrm{Si}_{2}$ with $M=\mathrm{Co}$, Ir and $x \leq 0.12$, grown in indium flux 
Fig. 2 (Color online)

Transversal magnetoresistivity $\rho(B)$ of $\mathrm{LuRh}_{2} \mathrm{Si}_{2}$ measured at $5 \mathrm{~K}$ with the field along the crystallographic $c$ direction. Solid lines represent quadratic and linear fits to the data below and above $1 \mathrm{~T}$, respectively

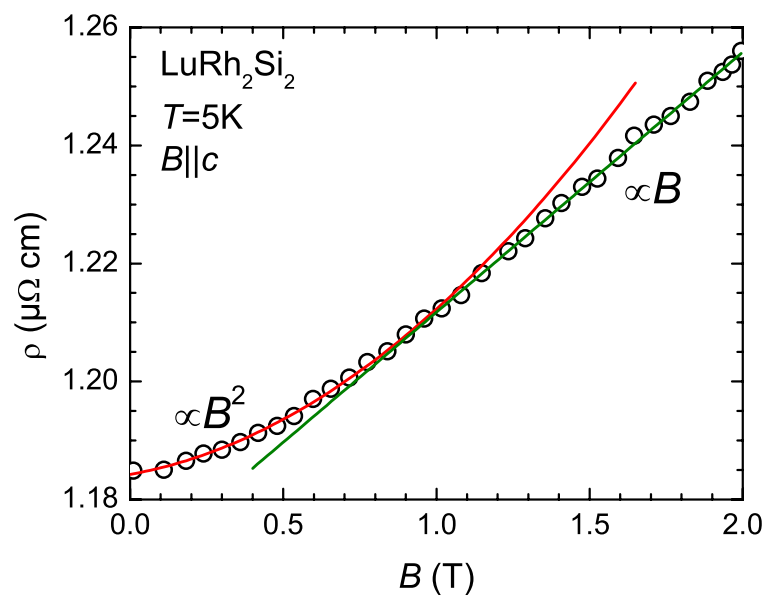

as described earlier [12]. The electric current flowed within the basal plane of the material, and the magnetic field was applied parallel to the current.

Measurements were also conducted on isostructural $\mathrm{LuRh}_{2} \mathrm{Si}_{2}$ (grown in indium flux as well) which served as a non-magnetic reference system. In this case, the transversal magnetoresistivity $(B \| c)$ was measured. The magnetoresistivity of $\mathrm{LuRh}_{2} \mathrm{Si}_{2}$ depicted in Fig. 2 displays a monotonic increase with increasing magnetic field. At small fields up to $1 \mathrm{~T}$ a quadratic form is observed which is the standard lowfield behavior for normal metals [22]. Above $1 \mathrm{~T}$ the resistivity increases linearly with increasing magnetic field which might arise from open orbits predicted by band structure calculations [23]. This reflects the simple, non-magnetic metallic nature of this material rendering it suitable as a reference compound. Therefore, the magnetoresistivity measured for a longitudinal orientation with the magnetic field perpendicular to the $c$-axis is expected to yield similar results as measured in a transversal setup. This is supported by a comparable evolution of the Hall coefficient for the different directions [24].

The $\rho(T)$ curve of $\mathrm{YbRh}_{2} \mathrm{Si}_{2}$ (not shown) exhibits a broad maximum at $T_{\max } \approx$ $80 \mathrm{~K}$. As was demonstrated [11] by careful resistivity and thermopower measurements on $\left(\mathrm{Lu}_{1-x} \mathrm{Yb}_{x}\right) \mathrm{Rh}_{2} \mathrm{Si}_{2}$ single crystals, this maximum reflects the transition between incoherent and coherent Kondo scattering of the charge carriers off $\mathrm{Yb}^{3+}$ whose effective $4 f$-state in the surrounding crystal-electric field (CEF) has a degeneracy greater than two. Upon volume compression by increasing the Lu content a second maximum at lower temperatures becomes apparent which is due to the substantial reduction of the Kondo temperature $T_{\mathrm{K}}$ of the CEF ground-state doublet. This second maximum assigned to $T_{\mathrm{K}}(x)$ allows an extrapolation to $x=1$ which revealed $T_{\mathrm{K}}=29 \mathrm{~K}$ for the pure compound, in good agreement with the temperature [12] at which the molar spin entropy yields $R \ln 2$.

Selected low-temperature curves of the magnetoresistivity in $\mathrm{YbRh}_{2} \mathrm{Si}_{2}$ are displayed in Fig. 3. With increasing magnetic field they exhibit first a pronounced crossover to smaller values (which is preceded by a maximum in $\rho(B)$ at low temperatures) and subsequently a linear increase extending beyond the scale used here. 
Fig. 3 (Color online) Magnetic field dependence of the resistivity $\rho$ for $\mathrm{YbRh}_{2} \mathrm{Si}_{2}$ at selected low temperatures from Ref. [13]. The magnetoresistivity was measured in a longitudinal setup with the field oriented perpendicular to the crystallographic $c$-direction. Arrows denote the position of the inflection points

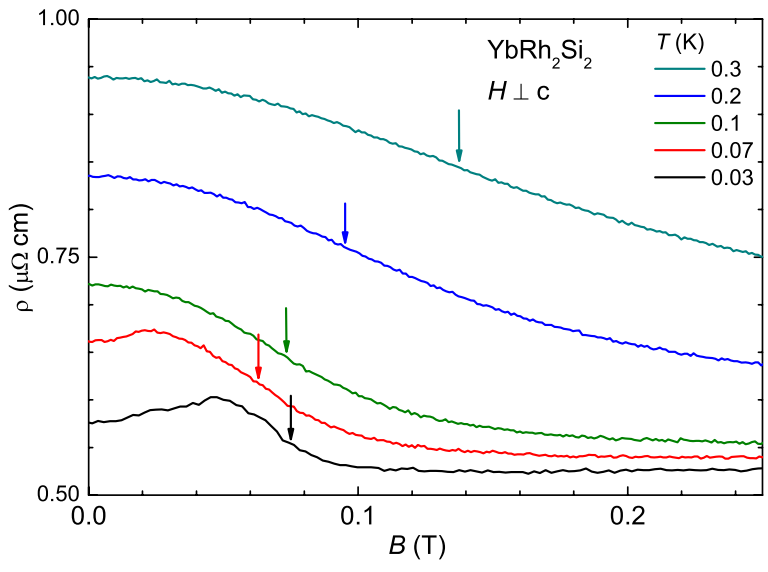

Such a crossover is typical for incoherent Kondo systems as an increasing magnetic field suppresses the underlying spin-flip scattering of the single-ion Kondo effect. For the case of the Kondo lattice relevant here, however, also different forms like a solely positive magnetoresistivity may occur as, e.g., observed in $\mathrm{YbIr}_{2} \mathrm{Si}_{2}$ [25]. In order to understand the two regimes at low and high fields in the paramagnetic state $\left(T>T_{\mathrm{N}}\right)$ of $\mathrm{YbRh}_{2} \mathrm{Si}_{2}$, one should consider its $B-T$ phase diagram. Like in $\mathrm{YbIr}_{2} \mathrm{Si}_{2}$, a Fermi-liquid phase formed by the heavy quasiparticles is present at high fields. In this regime the positive magnetoresistivity is in accordance with Fermiliquid theory. The pronounced crossover at low fields ( $c f$. arrows in Fig. 3) on the other hand is attributed to the quantum criticality of $\mathrm{YbRh}_{2} \mathrm{Si}_{2}$. This can be seen by the fact that the $T$-dependence of the position of the crossover as quantified by its inflection point extrapolates to the QCP [13]. At the lowest temperatures, however, an additional feature emerges. In contrast to the curves at higher temperatures, below $T_{\mathrm{N}}$ an increase of the resistivity with increasing magnetic field precedes the crossover. This increase is proportional to $B^{2}$ and is limited to the ordered phase where the temperature dependence of $\rho$ is also quadratic [16]. Both these signatures are in agreement with a Fermi-liquid picture. Remarkably, this form of the magnetoresistivity appears to be more sensitive to sample quality compared to the quadratic temperature dependence of the resistivity: For samples with twice the residual resistivity no such upturn is observed in $\rho(B)$ whereas the quadratic form of $\rho(T)$ is still present [26].

The phase diagram in Fig. 4 displays the evolution of the inflection point of the magnetoresistivity crossover with temperature. For $\mathrm{YbRh}_{2} \mathrm{Si}_{2}$ and within the low temperature range investigated here, the crossover shifts linearly towards lower fields upon cooling ( $c f$. solid line in Fig. 4). Below $100 \mathrm{mK}$, deviations from this linearity towards higher fields are observed: At the lowest temperature, the inflection point is shifted to higher fields. This non-monotonic evolution is attributed to the nearby transition into the antiferromagnetically ordered state as will be explained in the following from results on samples with isoelectronic substitution. The range at higher temperatures will be discussed along with susceptibility results in Sect. 3. 


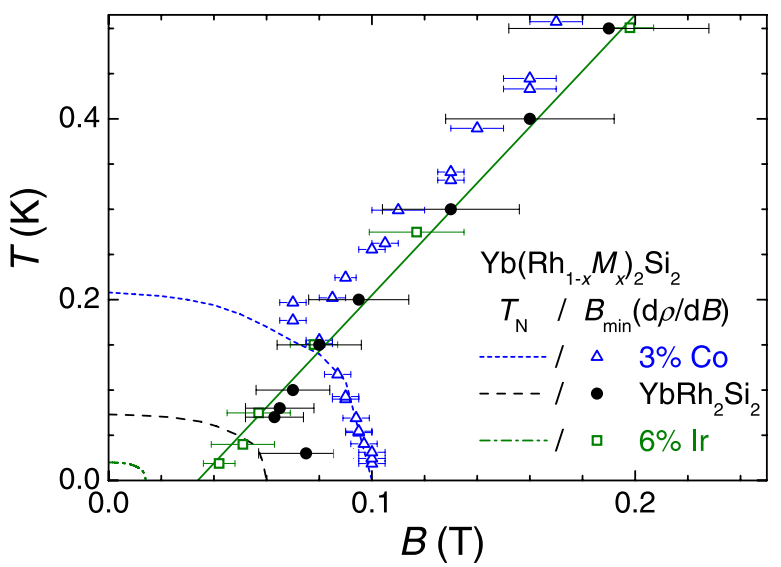

Fig. 4 (Color online) $T-B$ phase diagram of pure and substituted $\mathrm{YbRh}_{2} \mathrm{Si}_{2}$. Data sets represent the positions of the inflection point of the magnetoresistivity crossover for $\mathrm{Yb}\left(\mathrm{Rh}_{0.94} \mathrm{Ir}_{0.06}\right)_{2} \mathrm{Si}_{2}, \mathrm{YbRh}_{2} \mathrm{Si}_{2}$ and $\mathrm{Yb}\left(\mathrm{Rh}_{0.97} \mathrm{Co}_{0.03}\right)_{2} \mathrm{Si}_{2}$. Dashed lines display the boundaries of the antiferromagnetically ordered phase for these three compounds as derived from $\rho(T)$ and $\chi_{\mathrm{ac}}(T)$. The solid line represents a fit to the data on $\mathrm{Yb}\left(\mathrm{Rh}_{0.94} \mathrm{Ir}_{0.06}\right)_{2} \mathrm{Si}_{2}$ which agrees within the error with a fit to the data on $\mathrm{YbRh}_{2} \mathrm{Si}_{2}$ above $100 \mathrm{mK}$. Data for $\mathrm{YbRh}_{2} \mathrm{Si}_{2}$ are taken from Ref. [13] where points below $100 \mathrm{mK}$ were added as determined in Fig. 3

The width of the magnetoresistivity crossover is another important characteristic. As can be seen from Fig. 3, the crossover narrows as the temperature is lowered. In order to analyze this quantitatively, the full-width at half-maximum (FWHM) of the minimum in the derivative $\mathrm{d} \rho / \mathrm{dB}$ was determined, $c f . \mathrm{d} \rho / \mathrm{dB}$ at $0.1 \mathrm{~K}$ in the inset of Fig. 5. The FWHM values determined in this way at different temperatures are displayed in the main panel of Fig. 5 for different samples. Due to peculiarities arising in the proximity of the transition into the antiferromagnetically ordered phase, the data are limited to temperatures above $T_{\mathrm{N}}$. For $\mathrm{YbRh}_{2} \mathrm{Si}_{2}$, the width decreases monotonically upon reducing the temperature, and extrapolates to zero in the limit of zero temperature. As already deduced for the Hall coefficient, this does imply that the magnetoresistivity crossover sharpens to a discontinuous jump at the QCP. It is worth mentioning that in the calculation performed for a single-ion Kondo model [27] the magnetoresistivity behaves in a similar fashion, but with a finite FWHM at $T=0$ reflecting the finite Kondo energy scale. Consequently, the experimental observation of a vanishing width adopts a key role in the interpretation of the magnetoresistivity crossover.

Taking into account the observations listed above, we might conclude that (i) the magnetoresistivity crossover is linked to the quantum criticality and that (ii) such a crossover sharpens with decreasing temperature and extrapolates to a discontinuous jump in the limit of zero temperature. Consequently, it suggests, together with the analogous crossover of the Hall coefficient [14], that the Fermi surface reconstructs at the QCP. In fact, calculations performed within the Kondo-lattice model [19] suggest a similar behavior of the two magnetotransport properties.

Having identified the magnetoresistivity crossover as a signature of the Kondo breakdown in pure $\mathrm{YbRh}_{2} \mathrm{Si}_{2}$ we will use it henceforth as a probe to investigate the 


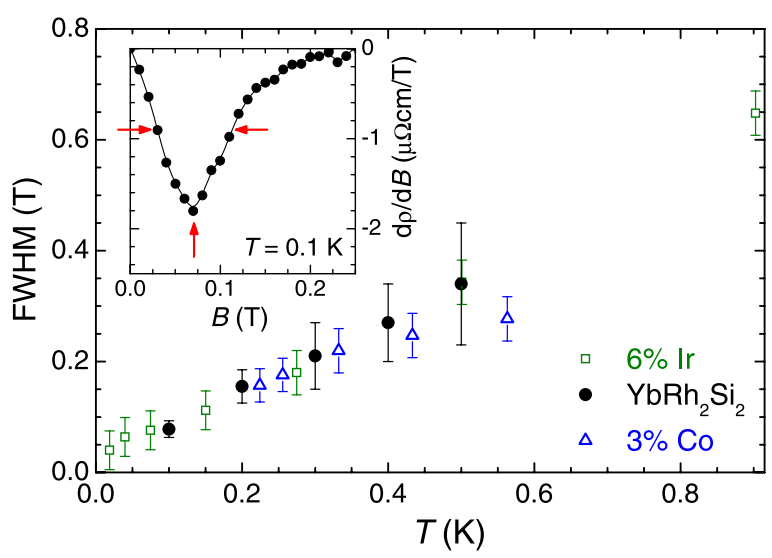

Fig. 5 (Color online) Full-width at half-maximum (FWHM) as derived from the derivate of the magnetoresistivity curves. For $\mathrm{YbRh}_{2} \mathrm{Si}_{2}$ and $\mathrm{Yb}\left(\mathrm{Rh}_{0.97} \mathrm{Co}_{0.03}\right)_{2} \mathrm{Si}_{2}$ the range is limited to above the Néel temperature of $0.07 \mathrm{~K}$ and $0.21 \mathrm{~K}$, respectively. Error bars of $\mathrm{Yb}\left(\mathrm{Rh}_{0.94} \mathrm{Ir}_{0.06}\right)_{2} \mathrm{Si}_{2}$ are larger due to the substitution-induced disorder. Inset shows the derivative $\mathrm{d} \rho / \mathrm{dB}$ for the example of pure $\mathrm{YbRh}_{2} \mathrm{Si}_{2}$ at $0.1 \mathrm{~K}$ and illustrates the determination of the FWHM (horizontal arrows) and the inflection point (vertical arrow)

effect of substitution. The isoelectronic substitution of rhodium by either iridium or cobalt gives rise to a change of the unit-cell volume. Smaller cobalt on the one hand induces a reduction of the volume: $3 \%$ of Co leads to a compression of $0.2 \%$. This converts to a pressure of $0.4 \mathrm{GPa}$, if the bulk modulus of $\mathrm{YbRh}_{2} \mathrm{Si}_{2}$ is taken into consideration [28]. On the other hand, the larger iridium expands the unit cell. However, the effect is less pronounced: A concentration of $6 \%$ iridium induces a volume increase of $0.03 \%$ which corresponds to a negative pressure of only $-0.06 \mathrm{GPa}$. The advantage of chemical pressure is based exactly on this ability to compress and expand the unit cell.

For $\mathrm{Yb}$ systems, the magnetism is expected to be stabilized with increasing (positive) pressure. This is opposite to the behavior of Ce systems. The major effect is caused by the differences in the ionic volumes of the two relevant $\mathrm{Yb}$ configurations [29]: The magnetic trivalent configuration is smaller compared to the non-magnetic divalent one. As a consequence, a smaller unit-cell volume stabilizes the magnetic configuration. In fact, both the application of external pressure and Co substitution, i.e. chemical pressure, lead to an increase of the Néel temperature in $\mathrm{YbRh}_{2} \mathrm{Si}_{2}[21$, 28]. The analogy of the evolution of $T_{\mathrm{N}}$ under hydrostatic and chemical pressure suggests that the volume effect predominates in the latter case. Also, the evolution of the magnetism under negative chemical pressure induced by the iridium substitution is in accord with the above described behavior: Here, the Néel temperature is found to be strongly suppressed. For example, by a substitution of $6 \%$ iridium, the magnetic transition is suppressed below the experimental limit of $20 \mathrm{mK}$. Importantly, for this sample the effect of negative chemical pressure can be reversed by applying hydrostatic pressure, which results in a recovery of the magnetic transition. Its evolution than follows that of the stoichiometric compound under pressure [30]. 
Fig. 6 (Color online) Magnetic field dependence of the resistivity $\rho$ for $\mathrm{Yb}\left(\mathrm{Rh}_{0.97} \mathrm{Co}_{0.03}\right)_{2} \mathrm{Si}_{2}$ at different temperatures. Arrows indicate the inflection point

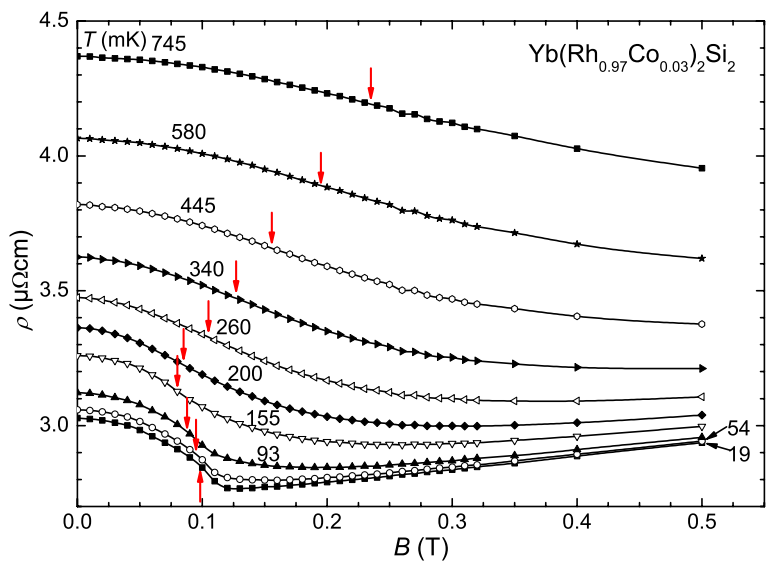

An important question arising from these studies concerns the evolution of the energy scales (observed in the magnetic field-temperature phase diagram) with chemical pressure, as was recently examined by a comprehensive study of the transport and magnetic properties [21]. In the present paper we focus in more detail on the results of the magnetoresistivity and the susceptibility.

For a substitution with $3 \%$ cobalt, the Néel temperature is increased to $T_{\mathrm{N}}=$ $0.21 \mathrm{~K}$. Figure 6 shows the magnetoresistivity of $\mathrm{Yb}\left(\mathrm{Rh}_{0.97} \mathrm{Co}_{0.03}\right)_{2} \mathrm{Si}_{2}$ at different temperatures. A characteristic crossover towards smaller values with increasing field is observed, similar to the one found for the pure compound. Furthermore, the increase of the magnetoresistivity at higher fields is also visible, again being reminiscent of the findings on $\mathrm{YbRh}_{2} \mathrm{Si}_{2}$. However, there are remarkable differences between the results obtained on the stoichiometric compound and those on $\mathrm{Yb}\left(\mathrm{Rh}_{0.97} \mathrm{Co}_{0.03}\right)_{2} \mathrm{Si}_{2}$ : (i) For the latter, the shoulder of the crossover steepens at temperatures below $T_{\mathrm{N}}$, in comparison to $\mathrm{YbRh}_{2} \mathrm{Si}_{2}$ in Fig. 3. (ii) No increase of the resistivity with increasing field is observed within the ordered phase, in contrast to the pure compound. This might be due to the enhanced disorder of the Co substituted sample, in line with what has been found for pure $\mathrm{YbRh}_{2} \mathrm{Si}_{2}$ samples of lesser quality, as discussed above. From observation (i) we derive a non-monotonic evolution of the crossover position as a function of temperature ( $c f$. arrows in Fig. 6). It is very interesting to compare the dependence of the $\rho(B)$-crossover for the $3 \%$ Co-substituted sample with that of pure $\mathrm{YbRh}_{2} \mathrm{Si}_{2}$. As shown in Fig. 4, at $T>0.2 \mathrm{~K}$ the crossover for the stoichiometric compound also follows a linear decrease with decreasing temperature. However, at lower temperatures the center of the crossover turns over and shifts to higher fields upon further cooling. At the lowest temperatures, the crossover position follows very precisely the phase boundary, $T_{\mathrm{N}}(B)$, extracted independently from $\rho(T)$ and $\chi_{\mathrm{ac}}(T)$. Finally, the position of the $\rho(B)$ crossover converges to the critical field $B_{\mathrm{N}}$ of the ordered phase as $T \rightarrow 0$. The reversed trend of the magnetoresistivity inflection point in the phase diagram in Fig. 4 found for $\mathrm{Yb}\left(\mathrm{Rh}_{0.97} \mathrm{Co}_{0.03}\right)_{2} \mathrm{Si}_{2}$ separates two regimes: At temperatures above $T_{\mathrm{N}}$ the crossover position matches the observations on the stoichiometric compound above its Néel temperature. Here, it was concluded to reflect the energy scale $T^{\star}$ of the Kondo breakdown. By contrast, 
Fig. 7 (Color online) Magnetic field dependence of the resistivity $\rho$ for $\mathrm{Yb}\left(\mathrm{Rh}_{0.94} \mathrm{Ir}_{0.06}\right)_{2} \mathrm{Si}_{2}$. Arrows indicate the position of the inflection point

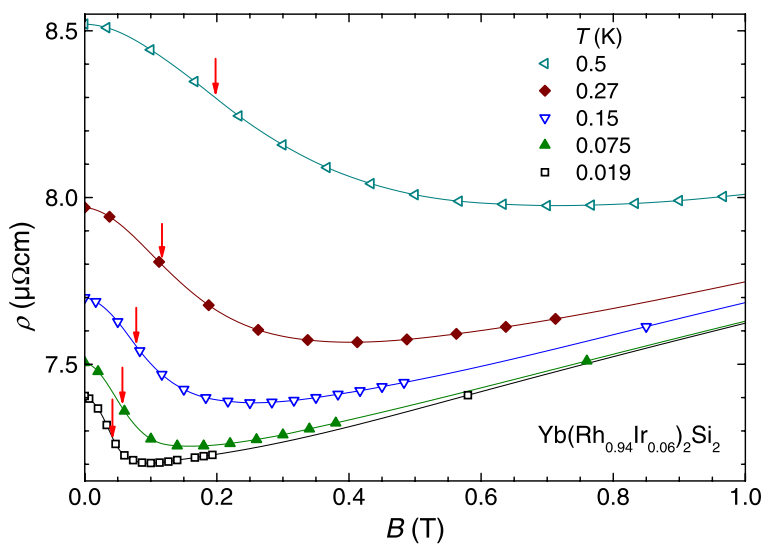

below $T_{\mathrm{N}}$ where the $\rho(B)$ crossover is locked to the boundary of the magnetically ordered phase, the classical transition dominates. Consequently, only the data above $T_{\mathrm{N}}$ can be used to extract information on the Kondo breakdown.

Taking into account the strong influence of the transition into the magnetically ordered phase on the behavior of the crossover seen in $\mathrm{Yb}\left(\mathrm{Rh}_{0.97} \mathrm{Co}_{0.03}\right)_{2} \mathrm{Si}_{2}$ we may re-examine the results on pure $\mathrm{YbRh}_{2} \mathrm{Si}_{2}$ : In both compounds and below the respective Néel temperature the magnetoresistivity crossover appears to be pinned to the border line of the antiferromagnetically ordered phase as already observed for higher Co substitutions. A more detailed future investigation is required to establish to which extent this behavior has some relevance for the quantum criticality in stoichiometric $\mathrm{YbRh}_{2} \mathrm{Si}_{2}$. Note that only one data point below $T_{\mathrm{N}}$ is presently available for the pure compound (cf. Fig. 4).

The restriction to temperatures above $T_{\mathrm{N}}$ applies also to the analysis of the width of the crossover which is therefore, in case of $\mathrm{Yb}\left(\mathrm{Rh}_{0.97} \mathrm{Co}_{0.03}\right)_{2} \mathrm{Si}_{2}, \mathrm{lim}$ ited to temperatures above $0.21 \mathrm{~K}$. In this temperature range the FWHM of $\mathrm{Yb}\left(\mathrm{Rh}_{0.97} \mathrm{Co}_{0.03}\right)_{2} \mathrm{Si}_{2}$ again resembles the behavior observed for $\mathrm{YbRh}_{2} \mathrm{Si}_{2}$ up to $0.35 \mathrm{~K}$, whereas at higher temperatures somewhat lower FWHM values were observed for the Co substituted sample ( $c f$. Fig. 5).

Since both $T_{\mathrm{N}}$ and the temperature below which the magnetoresistivity crossover deviates from its high-temperature linearity are increased in $\mathrm{Yb}\left(\mathrm{Rh}_{0.97} \mathrm{Co}_{0.03}\right)_{2} \mathrm{Si}_{2}$, i.e., for positive chemical pressure, the application of negative chemical pressure by iridium substitution may unfold an extended field range of the crossover position in $\rho(B)$. This is, in fact, inferred from the magnetoresistivity data for $\mathrm{Yb}\left(\mathrm{Rh}_{0.94} \mathrm{Ir}_{0.06}\right)_{2} \mathrm{Si}_{2}$ shown in Fig. 7. For this sample, the magnetic transition takes place just below $20 \mathrm{mK}$. Again, $\rho(B)$ exhibits a crossover as observed in $\mathrm{YbRh}_{2} \mathrm{Si}_{2}$. Also, the increasing magnetoresistivity at elevated field is reproduced. Importantly, the inflection point of the crossover shifts to lower fields as the temperature is decreased, with this trend now continuing to lowest temperatures. The evolution of the position of the magnetoresistivity crossover is demonstrated in the magnetic fieldtemperature phase diagram in Fig. 4. At temperatures above $100 \mathrm{mK}$, the data match with those of $\mathrm{YbRh}_{2} \mathrm{Si}_{2}$ as well as of the $3 \%$ Co substituted material. In particular, 
they follow the linear form observed for the pure compound within this temperature range. The main difference appears at $T \lesssim 100 \mathrm{mK}$. Whereas for $\mathrm{YbRh}_{2} \mathrm{Si}_{2}$ deviations from linearity set in when the border of the antiferromagnetically ordered phase is approached, the proportionality is observed in $\mathrm{Yb}\left(\mathrm{Rh}_{0.94} \mathrm{Ir}_{0.06}\right)_{2} \mathrm{Si}_{2}$ down to $19 \mathrm{mK}$, the lowest temperature of our experiment. This can be quantified: Fits of a linear form to the whole data set of $\mathrm{Yb}\left(\mathrm{Rh}_{0.94} \mathrm{Ir}_{0.06}\right)_{2} \mathrm{Si}_{2}$ and to the data of $\mathrm{YbRh}_{2} \mathrm{Si}_{2}$ in the range above $100 \mathrm{mK}$ yield identical parameters within the experimental error. Both fits extrapolate to an intersect with the abscissa at a field of $B^{\star}=35(5) \mathrm{mT}$. Surprisingly, this critical field agrees well with that at which the $A(B)$-coefficient of $\Delta \rho(T, B)=A(B) \cdot T^{2}$ diverges for $\mathrm{Yb}\left(\mathrm{Rh}_{0.94} \mathrm{Ir}_{0.06}\right)_{2} \mathrm{Si}_{2}[21]$.

The difference of $\mathrm{Yb}\left(\mathrm{Rh}_{0.94} \mathrm{Ir}_{0.06}\right)_{2} \mathrm{Si}_{2}$ compared to $\mathrm{YbRh}_{2} \mathrm{Si}_{2}$ clearly lies in the fact that the magnetic transition temperature is decreased in the former material. Likewise, the critical field is expected to be smaller. For an estimate we scale the magnetic phase boundary deduced for the stoichiometric system with the reduced magnetic temperature of $\mathrm{Yb}\left(\mathrm{Rh}_{0.94} \mathrm{Ir}_{0.06}\right)_{2} \mathrm{Si}_{2}$. Employing the upper limit of $T_{\mathrm{N}}=20 \mathrm{mK}$ yields an upper limit of the critical field of $B_{\mathrm{N}} \lesssim 15 \mathrm{mT}$. Consequently, the magnetoresistivity crossover at $35 \mathrm{mT}$ is well separated from the magnetic phase even at lowest temperatures. Taking the observations on the whole substitution series together, it is suggestive that the linearity of the $\rho(B)$-crossover may reflect the behavior intrinsic to quantum criticality, while the vicinity of the classical phase transition seems to cause deviations from this behavior.

Following the concepts outlined above, the width of the magnetoresistivity crossover should follow a global trend to lowest temperatures as well. In fact, the FWHM in $\mathrm{Yb}\left(\mathrm{Rh}_{0.94} \mathrm{Ir}_{0.06}\right)_{2} \mathrm{Si}_{2}$ is found to decrease as the temperature is lowered in line with the behavior observed in $\mathrm{YbRh}_{2} \mathrm{Si}_{2}$ above its Néel temperature (see Fig. 5). However, in this case the width of the $\rho(B)$-crossover appears to be substantially enlarged due to the unavoidable disorder introduced by the Ir-substitution. Within the error bars in Fig. 5 the FWHM of $\mathrm{Yb}\left(\mathrm{Rh}_{0.94} \mathrm{Ir}_{0.06}\right)_{2} \mathrm{Si}_{2}$ may well be extrapolated to zero as $T \rightarrow 0$.

\section{Susceptibility}

As a complementary probe we now discuss results of a.c. susceptibility, $\chi_{\mathrm{ac}}$, measurements. The susceptibility is sensitive to both the magnetic transition and the $T^{\star}(B)$ energy scale [13]: For $\mathrm{YbRh}_{2} \mathrm{Si}_{2}, \chi_{\mathrm{ac}}(T)$ was found to exhibit a kink when entering the magnetically ordered phase as well as a broad maximum reflecting the position of the Kondo-breakdown crossover.

The temperature dependences of the susceptibility for $\mathrm{Yb}\left(\mathrm{Rh}_{0.975} \mathrm{Ir}_{0.025}\right)_{2} \mathrm{Si}_{2}$ and $\mathrm{Yb}\left(\mathrm{Rh}_{0.97} \mathrm{Co}_{0.03}\right)_{2} \mathrm{Si}_{2}$ are displayed in Fig. 8. The phase transition is clearly observed in both compounds in the absence of a magnetic field at $T_{\mathrm{N}}=40 \mathrm{mK}$ (see inset) and $175 \mathrm{mK}$, respectively. As expected, the application of a magnetic field suppresses the transition temperature. From the evolution of the transition in the magnetic fieldtemperature phase diagram, Fig. 9, critical fields of $B_{\mathrm{N}}=90 \mathrm{mT}$ (3\% Co substituted) and $35 \mathrm{mT}$ ( $2.5 \%$ Ir substituted) are deduced. We note that the critical field deduced from resistivity measurements on $\mathrm{Yb}\left(\mathrm{Rh}_{0.97} \mathrm{Co}_{0.03}\right)_{2} \mathrm{Si}_{2}$ is slightly higher ( $c f$. Fig. 3). 


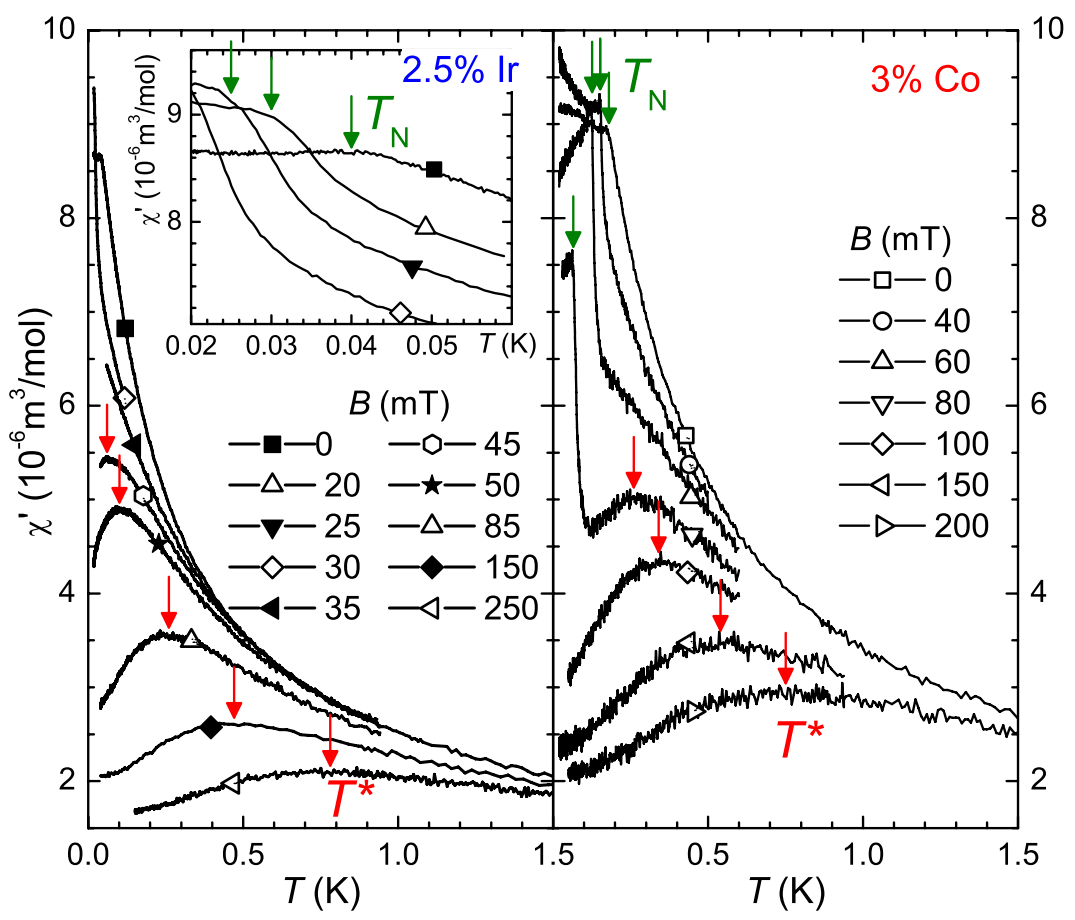

Fig. 8 (Color online) Temperature dependence of the a.c. susceptibility for $\mathrm{Yb}\left(\mathrm{Rh}_{0.975} \operatorname{Ir}_{0.025}\right)_{2} \mathrm{Si}_{2}($ left panel) and $\mathrm{Yb}\left(\mathrm{Rh}_{0.97} \mathrm{Co}_{0.03}\right)_{2} \mathrm{Si}_{2}$ (right panel). Green and red arrows denote the positions of the Néel temperature and the maximum in $\chi_{\mathrm{ac}}(T)$, respectively. The latter is associated with the $T^{\star}(B)$ scale

Fig. 9 (Color online) Phase diagram of

$\mathrm{Yb}\left(\mathrm{Rh}_{0.975} \mathrm{Ir}_{0.025}\right)_{2} \mathrm{Si}_{2}$ and $\mathrm{Yb}\left(\mathrm{Rh}_{0.97} \mathrm{Co}_{0.03}\right)_{2} \mathrm{Si}_{2}$ derived from $\chi_{\text {ac-measurements. }}$

Triangles denote the transition temperature of the magnetically ordered phase as marked in Fig. 8. Circles/squares represent the position of the maximum in $\chi_{\text {ac }}(T)$

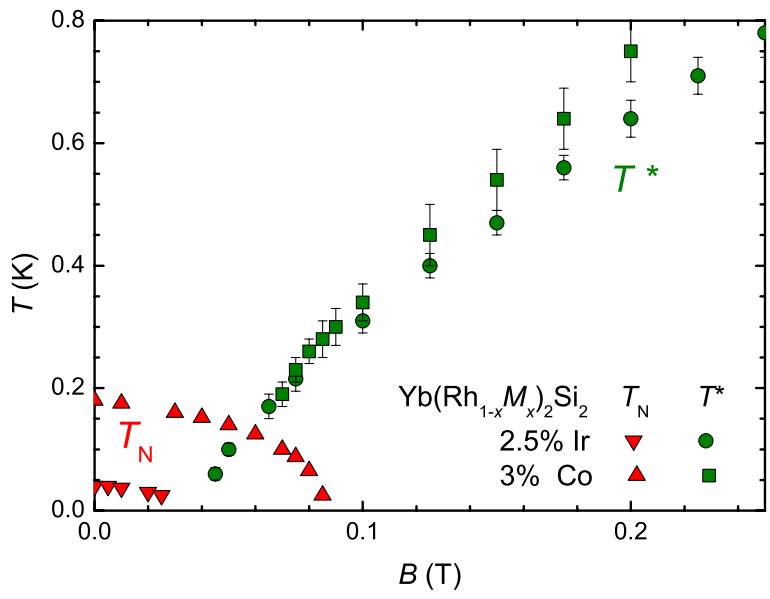

Also, the exact values of $B_{\mathrm{N}}$ depend to some extend on the extrapolation used in $T_{\mathrm{N}}(B) \rightarrow 0$.

The maximum in $\chi_{\mathrm{ac}}(T)$ reflecting the position of the Kondo-breakdown energy scale $T^{\star}(B)$ is present in both systems for fields above $45 \mathrm{mT}$ and $60 \mathrm{mT}$, respec- 
Fig. 10 (Color online)

Zero-temperature phase diagram based on Ref. [21]. The critical field $B_{\mathrm{N}}$ of the

antiferromagnetically ordered phase and the critical field of the $T^{\star}(B)$-line are depicted as a function of iridium and cobalt substitution. The values for $\mathrm{YbRh}_{2} \mathrm{Si}_{2}$ were taken from Ref. [13]

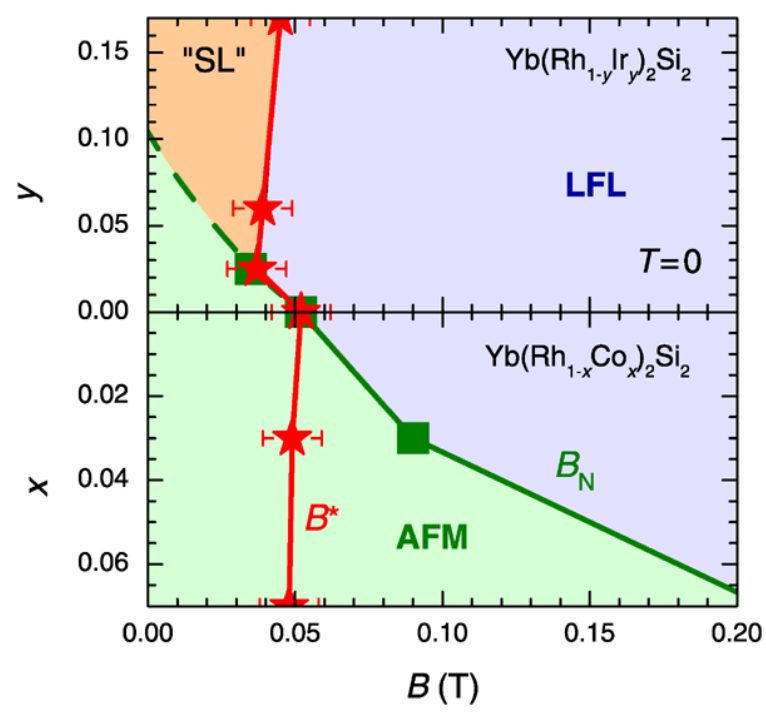

tively. With increasing magnetic field, the position of these maxima is shifted to higher temperatures as shown in Fig. 9. Although the $T^{\star}(B)$-lines extracted from susceptibility and magnetoresistivity are not located at exactly the same position of the $B-T$ phase diagram [2], within the error bars they follow the same trend ( $c f$. Fig. 4). However, the susceptibility results allow to cover a larger temperature range mainly because the respective feature in magnetoresistivity becomes very broad at these higher temperatures ( $c f$. Fig. 3). Here we also note that the magnetoresistivity was measured at constant temperatures whereas the temperature was swept at constant magnetic field for susceptibility measurements, a fact that is instrumental for the issue at hand. The extended temperature range of the phase diagram Fig. 9 (compared to the magnetoresistivity) reveals that the $T^{\star}(B)$-line extracted from susceptibility obeys a sublinear behavior. In general, the comparison of the $T^{\star}(B)$-line extracted from the different quantities as shown in Fig. 1 already appears to indicate different forms: For magnetoresistivity, Hall resistivity, and magnetostriction an almost linear $T^{\star}(B)$ behavior is seen above $150 \mathrm{mK}$, whereas magnetization and susceptibility seem to exhibit a sublinear one. More precise measurements over an extended temperature range would be helpful to clarify this in more detail. Again, the robustness of the $T^{\star}(B)$-line for the different substitutions is striking. Finally, the extrapolated critical fields at which $T^{\star} \rightarrow 0$ yield very similar values of $B^{\star}=37 \mathrm{mT}$ and $50 \mathrm{mT}$ for $\mathrm{Yb}\left(\mathrm{Rh}_{0.975} \mathrm{Ir}_{0.025}\right)_{2} \mathrm{Si}_{2}$ and $\mathrm{Yb}\left(\mathrm{Rh}_{0.97} \mathrm{Co}_{0.03}\right)_{2} \mathrm{Si}_{2}$, respectively.

The strength of the susceptibility results is based on the fact that it appears to allow a more unaffected view on $T^{\star}(B)$ : For a sample with $7 \%$ cobalt substitution, the signatures of the Kondo-breakdown crossover were even observed [21] within the antiferromagnetically ordered phase below $T_{\mathrm{N}}=410 \mathrm{mK}$. On the other hand, for $3 \%$ Co-substitution, the low $T_{\mathrm{N}}=90 \mathrm{mK}$ does not allow for a $\chi_{\mathrm{ac}}(T)$ maximum to fully develop inside the magnetically ordered phase.

Making use of all susceptibility results on the different samples we can construct a zero-temperature phase diagram, illustrating the evolution of the two critical fields 
(see Fig. 10). Here, it is easily seen that the critical field $B_{\mathrm{N}}$ of the antiferromagnetic phase increases as the unit cell shrinks (from Ir substitution via the pure $\mathrm{YbRh}_{2} \mathrm{Si}_{2}$ towards increasing Co substitution). By contrast, the critical field $B^{\star}$ of the $T^{\star}(B)$ line stays almost constant. As a consequence the initial coincidence of the two critical fields $B_{\mathrm{N}}$ and $B^{\star}$ observed in pure $\mathrm{YbRh}_{2} \mathrm{Si}_{2}$ is lifted: For the case of $6 \%$ iridium substitution antiferromagnetism has become weaker and is found only below $T_{\mathrm{N}}=$ $20 \mathrm{mK}$ and $B_{\mathrm{N}} \leq 15 \mathrm{mT}$ without the immediate recovery of a Fermi liquid in an extended parameter range at $T>T^{\star}$ and $B_{\mathrm{N}}<B<B^{\star}$. Here, the pressing question at hand concerns the fate of the magnetic moments: On the one hand, they appear not to order and, on the other hand, they cannot be fully screened, as this only takes place at fields larger than $B^{\star}$. Consequently, one may speculate that they form a new type of metallic spin-liquid phase. This could arise, for instance, from frustration between different magnetic interactions [31].

For samples with cobalt substitution, the magnetic phase extends into the regime, where the Kondo effect is intact. Here, the composite quasiparticles appear to be solidly formed and, consequently, the magnetism ought to be of itinerant nature. This is, in fact, supported [21], e.g., by analyzing the shape of the phase boundary $T_{\mathrm{N}}(B)$. It is reassuring to note that magnetization measurements on $\mathrm{YbRh}_{2} \mathrm{Si}_{2}$ under hydrostatic pressure [32] indeed confirmed the positions of both $T_{\mathrm{N}}(B)$ and $T^{\star}(B)$ as deduced for the Co-substituted samples.

The most interesting part of the phase diagram is located close to the stoichiometric $\mathrm{YbRh}_{2} \mathrm{Si}_{2}$ : Remarkably, there, $B^{\star}$ and $B_{\mathrm{N}}$ seem to coincide in a finite range, in agreement with theoretical predictions within the locally critical scenario [33, 34]. Although our magnetoresistivity and susceptibility results indicate that this coincidence might extend towards slightly Ir-substituted samples, the question arises why this situation is realized around the stoichiometric compound. Of course, it should also be investigated whether $B_{\mathrm{N}} \approx B^{\star}$ holds for $\mathrm{Yb}\left(\mathrm{Rh}_{1-x} \mathrm{Co}_{x}\right)_{2} \mathrm{Si}_{2}$ samples with $x<3 \%$, too: As pointed out in Sect. 2 the effect of Co substitution is much more pronounced in comparison to Ir substitution. We conclude this chapter by stating that within the experimental error the $B_{\mathrm{N}^{-}}$and $B^{\star}$-lines in the zero-temperature phase diagram might coincide within an extended range of substitution, from stoichiometric $\mathrm{YbRh}_{2} \mathrm{Si}_{2}$ to $3 \%$ iridium concentration ( $c f$. Fig. 10). We thus suggest that an in-depth study will help to clarify this central point about the understanding of quantum criticality in $\mathrm{YbRh}_{2} \mathrm{Si}_{2}$.

Our study does not reveal any indication of a valence transition as the underlying mechanism of the quantum criticality: First, a valence transition at $p_{\mathrm{v}}^{\mathrm{c}}$ and $T=0$ in stoichiometric $\mathrm{YbRh}_{2} \mathrm{Si}_{2}$ as speculated in Ref. [35] should become apparent in our study of $\mathrm{YbRh}_{2} \mathrm{Si}_{2}$ under positive and negative chemical pressure. Second, no signature of a first order transition appears under pressure as expected for a critical end point of a valence transition that might give rise to a QCP [36]. In addition, this absence of a first order transition seems to rule out a quantum tricritical point as the origin of the quantum criticality [37]. 


\section{Conclusions}

In this paper we have emphasized the unconventional nature of the quantum criticality in $\mathrm{YbRh}_{2} \mathrm{Si}_{2}$. Discontinuities of the Hall coefficient and the magnetoresistivity in the extrapolation to the QCP at zero temperature suggest an abrupt jump in the Fermi volume leading to a reconstruction of the Fermi surface, in accordance with a breakdown of the Kondo effect. This is expected in unconventional scenarios of heavy-fermion quantum criticality [7-10]. Very surprisingly, the antiferromagnetic instability appears to be decoupled from the Fermi surface reconstruction under the application of chemical pressure. For negative chemical pressure an intermediate phase with nonFermi-liquid behavior is observed which may turn out to be a new form of metallic spin-liquid. It is interesting to note that a non-Fermi-liquid phase at $T=0$ in an extended range of control parameter has been reported for other Yb-based heavyfermion compounds, i.e. YbAgGe [38] and Ge-substituted $\mathrm{YbRh}_{2} \mathrm{Si}_{2}$ for $B \| c$ [39] with magnetic field as control parameter, and $\beta-\mathrm{YbAlB}_{4}$ under applied pressure [40].

The most intriguing discovery made in the present investigation concerns the peculiar coincidence of the antiferromagnetic and the Kondo-breakdown instabilities in a finite substitution range of $\mathrm{Yb}\left(\mathrm{Rh}_{1-x} \mathrm{Ir}_{x}\right)_{2} \mathrm{Si}_{2}$ which confirms recent theoretical results obtained in the framework of local quantum criticality [33, 34].

Acknowledgements The authors acknowledge partial support by the DFG Research Group 960 'Quantum Phase Transitions'.

\section{References}

1. Special issue: Quantum criticality and novel phases (QCNP 2009). Phys. Status Solidi B 247, 457-764 (2010)

2. P. Gegenwart, Q. Si, F. Steglich, Quantum criticality in heavy-fermion metals. Nat. Phys. 4, 186-197 (2008)

3. S. Doniach, The Kondo lattice and weak antiferromagnetism. Physica B+C 91, 231-234 (1977)

4. J.A. Hertz, Quantum critical phenomena. Phys. Rev. B 14, 1165-1184 (1976)

5. A.J. Millis, Effect of a nonzero temperature on quantum critical-points in itinerant fermion systems. Phys. Rev. B 48, 7183-7196 (1993)

6. T. Moriya, T. Takimoto, Anomalous properties around magnetic instability in heavy electron systems. J. Phys. Soc. Jpn. 64, 960-969 (1995)

7. Q. Si, M.S. Rabello, K. Ingersent, J.L. Smith, Locally critical quantum phase transitions in strongly correlated metals. Nature 413, 804-808 (2001)

8. P. Coleman, C. Pépin, Q. Si, R. Ramazashvili, How do Fermi liquids get heavy and die? J. Phys., Condens. Matter 13, R723-R738 (2001)

9. T. Senthil, S. Sachdev, M. Vojta, Fractionalized Fermi liquids. Phys. Rev. Lett. 90, 216403 (2003)

10. C. Pépin, Kondo breakdown as a selective Mott transition in the Anderson lattice. Phys. Rev. Lett. 98, 206401 (2007)

11. U. Köhler, N. Oeschler, F. Steglich, S. Maquilon, Z. Fisk, Energy scales of $\mathrm{Lu}_{1-x} \mathrm{Yb}_{x} \mathrm{Rh}_{2} \mathrm{Si}_{2}$ by means of thermopower investigations. Phys. Rev. B 77, 104412 (2008)

12. O. Trovarelli, C. Geibel, S. Mederle, C. Langhammer, F.M. Grosche, P. Gegenwart, M. Lang, G. Sparn, F. Steglich, $\mathrm{YbRh}_{2} \mathrm{Si}_{2}$ : Pronounced non-Fermi-liquid effects above a low-lying magnetic phase transition. Phys. Rev. Lett. 85, 626-629 (2000)

13. P. Gegenwart, T. Westerkamp, C. Krellner, Y. Tokiwa, S. Paschen, C. Geibel, F. Steglich, E. Abrahams, Q. Si, Multiple energy scales at a quantum critical point. Science 315, 969-971 (2007)

14. S. Paschen, T. Lühmann, S. Wirth, P. Gegenwart, O. Trovarelli, C. Geibel, F. Steglich, P. Coleman, Q. $\mathrm{Si}$, Hall-effect evolution across a heavy-fermion quantum critical point. Nature 432, 881-885 (2004) 
15. P. Gegenwart, J. Custers, C. Geibel, K. Neumaier, T. Tayama, K. Tenya, O. Trovarelli, F. Steglich, Magnetic-field induced quantum critical point in $\mathrm{YbRh}_{2} \mathrm{Si}_{2}$. Phys. Rev. Lett. 89, 056402 (2002)

16. J. Custers, P. Gegenwart, H. Wilhelm, K. Neumaier, Y. Tokiwa, O. Trovarelli, C. Geibel, F. Steglich, C. Pépin, P. Coleman, The break-up of heavy electrons at a quantum critical point. Nature 424, 524527 (2003)

17. N. Tsujii, K. Yoshimura, K. Kosuge, Deviation from the Kadowaki-Woods relation in Yb-based intermediate-valence systems. J. Phys., Condens. Matter 15, 1993-2003 (2003)

18. A.C. Jacko, J.O. Fjaerestad, B.J. Powell, A unified explanation of the Kadowaki-Woods ratio in strongly correlated metals. Nat. Phys. 5, 422-425 (2009)

19. P. Coleman, J.B. Marston, A.J. Schofield, Transport anomalies in a simplified model for a heavyelectron quantum critical point. Phys. Rev. B 72, 245111 (2005)

20. S. Hartmann, N. Oeschler, C. Krellner, C. Geibel, S. Paschen, F. Steglich, Thermopower evidence for an abrupt Fermi surface change at the quantum critical point of $\mathrm{YbRh}_{2} \mathrm{Si}_{2}$. Phys. Rev. Lett. 104, 096401 (2010)

21. S. Friedemann, T. Westerkamp, M. Brando, N. Oeschler, S. Wirth, P. Gegenwart, C. Krellner, C. Geibel, F. Steglich, Detaching the antiferromagnetic quantum critical point from the Fermi-surface reconstruction in $\mathrm{YbRh}_{2} \mathrm{Si}_{2}$. Nat. Phys. 5, 465-469 (2009)

22. C.M. Hurd, The Hall Effect in Metals and Alloys (Plenum, New York, 1972)

23. S. Friedemann, N. Oeschler, C. Krellner, C. Geibel, S. Wirth, F. Steglich, S. Paschen, S. MaQuilon, Z. Fisk, G. Zwicknagl, Hall effect measurements on $\mathrm{YbRh}_{2} \mathrm{Si}_{2}$ in the light of electronic structure calculations. arXiv:0803.4428v1 (2008)

24. S. Friedemann, N. Oeschler, C. Krellner, C. Geibel, F. Steglich, Quantum criticality in $\mathrm{Yb}\left(\mathrm{Rh}_{0.97} \mathrm{Co}_{0.03}\right)_{2} \mathrm{Si}_{2}$ probed by low-temperature resistivity. J. Phys., Conf. Ser. 100, 012038 (2010)

25. F. Weickert, Quantenkritisches Verhalten in hochkorrelierten Elektronensystemen. Ph.D. dissertation, Technische Universität Dresden (2006)

26. J. Custers, Quantum critical behavior in the heavy fermion compounds $\mathrm{YbRh}_{2} \operatorname{Si}_{2}$ and $\mathrm{CeIn}_{3-x} \operatorname{Sn}_{x}$. Ph.D. dissertation, Technical University Dresden (2004)

27. P. Schlottmann, Bethe-ansatz solution of the ground-state of the $S U(2 j+1)$ Kondo (CoqblinSchrieffer) model: magnetization, magnetoresistance and universality. Z. Phys. B, Condens. Matter 51, 223-235 (1983)

28. S. Mederle, R. Borth, C. Geibel, F.M. Grosche, G. Sparn, O. Trovarelli, F. Steglich, An unconventional metallic state in $\mathrm{YbRh}_{2}\left(\mathrm{Si}_{1-x} \mathrm{Ge}_{x}\right)_{2}$-a high pressure study. J. Phys., Condens. Matter 14, 1073110736 (2002)

29. A.V. Goltsev, M.M. Abd-Elmeguid, Origin of the pressure dependence of the Kondo temperature in Ce- and Yb-based heavy-fermion compounds. J. Phys., Condens. Matter 17, S813-S821 (2005)

30. M.E. Macovei, M. Nicklas, C. Krellner, C. Geibel, F. Steglich, Effect of pressure and Ir substitution in $\mathrm{YbRh}_{2} \mathrm{Si}_{2}$. J. Phys., Condens. Matter 20, 505205 (2008)

31. P. Coleman, Quantum criticality and novel phases: a panel discussion. Phys. Status Solidi B 247, 506-512 (2010)

32. Y. Tokiwa, P. Gegenwart, C. Geibel, F. Steglich, Separation of energy scales in undoped $\mathrm{YbRh}_{2} \mathrm{Si}_{2}$ under hydrostatic pressure. J. Phys. Soc. Jpn. 78, 123708 (2009)

33. Q. Si, Global magnetic phase diagram and local quantum criticality in heavy fermion metals. Physica B 378, 23-27 (2006)

34. Q. Si, Quantum criticality and global phase diagram of magnetic heavy fermions. Phys. Status Solidi B 247, 457-764 (2010)

35. G. Knebel, R. Boursier, E. Hassinger, G. Lapertot, P.G. Niklowitz, A. Pourret, B. Salce, J.P. Sanchez, I. Sheikin, P. Bonville, H. Harima, J. Flouquet, Localization of $4 f$ state in $\mathrm{YbRh}_{2} \mathrm{Si}_{2}$ under magnetic field and high pressure: Comparison with $\mathrm{CeRh}_{2} \mathrm{Si}_{2}$. J. Phys. Soc. Jpn. 75, 114709 (2006)

36. S. Watanabe, A. Tsuruta, K. Miyake, J. Flouquet, Magnetic-field control of quantum critical points of valence transition. Phys. Rev. Lett. 100, 236401 (2008)

37. T. Misawa, Y. Yamaji, M. Imada, $\mathrm{YbRh}_{2} \mathrm{Si}_{2}$ : Quantum tricritical behavior in itinerant electron systems. J. Phys. Soc. Jpn. 77, 093712 (2008)

38. S.L. Bud'ko, E. Morosan, P.C. Canfield, Magnetic field induced non-Fermi-liquid behavior in YbAgGe single crystals. Phys. Rev. B 69, 014415 (2004)

39. J. Custers, P. Gegenwart, C. Geibel, F. Steglich, P. Coleman, S. Paschen, Evidence for a non-Fermiliquid phase in Ge-substituted $\mathrm{YbRh}_{2} \mathrm{Si}_{2}$. Phys. Rev. Lett. 104, 186402 (2010)

40. S. Nakatsuji, K. Kuga, Y. Machida, T. Tayama, T. Sakakibara, Y. Karaki, H. Ishimoto, S. Yonezawa, Y. Maeno, E. Pearson, G.G. Lonzarich, L. Balicas, H. Lee, Z. Fisk, Superconductivity and quantum criticality in the heavy-fermion system $\beta$-YbAlB 4 . Nat. Phys. 4, 603-607 (2008) 